\title{
Influence of Erbium-doped Fiber Amplifiers on the Timing Stability of Optical Pulse Trains
}

\author{
F. Löhl ${ }^{\dagger 1}$, V. Arsov ${ }^{1}$, M. Felber ${ }^{1}$, K. Hacker ${ }^{1}$, B. Lorbeer $^{1}$, F. Ludwig ${ }^{1}$, \\ K. Matthiesen ${ }^{1}$, J. Müller ${ }^{2}$, H. Schlarb ${ }^{1}$, B. Schmidt ${ }^{1}$, S. Schulz ${ }^{1}$, \\ A. Winter ${ }^{3}$, J. Zemella ${ }^{3}$ \\ ${ }^{1}$ Deutsches Elektronen Synchrotron (DESY), 22607 Hamburg, Germany \\ ${ }^{2}$ Technische Universität Hamburg-Harburg, 21071 Hamburg, Germany \\ ${ }^{3}$ Universität Hamburg, 22761 Hamburg, Germany \\ †florian.loehl@desy.de
}

\begin{abstract}
The influence of an erbium-doped fiber amplifier on the timing stability of an optical pulse train was characterized with balanced optical cross-correlation. Under optimized conditions, an added timing jitter of 0.5 fs was achieved.
\end{abstract}

(C) 2008 Optical Society of America

OCIS codes: (120.3940) Metrology; (320.7160) Ultrafast technoloy

Ultraviolet and X-ray free electron lasers like FLASH (Free electron LASer in Hamburg) or the planned European XFEL require synchronization of various devices in the accelerator to better than $10 \mathrm{fs}$ for experiments in a pumpprobe configuration. The most critical devices are the photo-injector laser, the RF gun, the accelerating modules in front of the bunch compressors, and pump-probe and seed lasers for user experiments. The locations are separated by $400 \mathrm{~m}$ for FLASH and by $3.5 \mathrm{~km}$ for the XFEL.

The high accuracy of synchronization required over such large distances cannot be achieved by conventional coaxial RF distribution systems. At FLASH and for the XFEL an optical synchronization system, developed in collaboration with MIT, will be implemented [1]. The system uses a mode-locked erbium-doped fiber laser operating in the soliton regime with a repetition rate of around $200 \mathrm{MHz}$ [2] which is locked to a microwave oscillator for improved lowfrequency stability. The synchronization information is contained in the precise repetition rate of the fiber laser. The high-frequency timing jitter of these lasers is crucial for the performance of the complete system, since it is not possible to reduce the timing jitter above frequencies of a few $\mathrm{kHz}$ by using a phase locked loop acting on a piezo driven mirror or a fiber stretcher for optical path length stabilization. The phase noise of the $200 \mathrm{MHz}$ soliton laser and a $40.8 \mathrm{MHz}$ stretched pulse laser is shown in Fig. 1. It amounts to values of around $10 \mathrm{fs}$ for both lasers, making either laser types a candidate for the master oscillator of the system.

a)

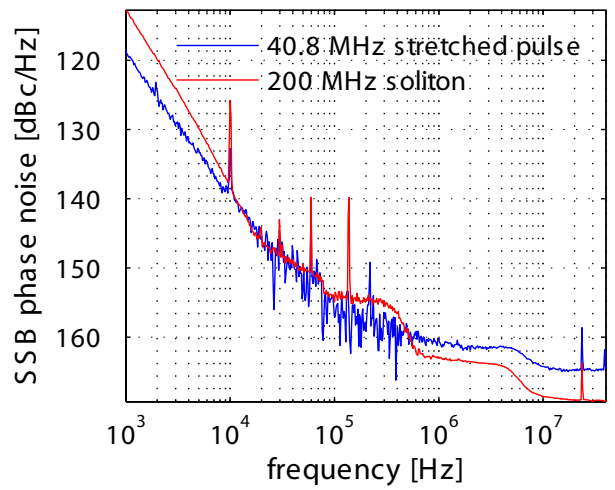

b)

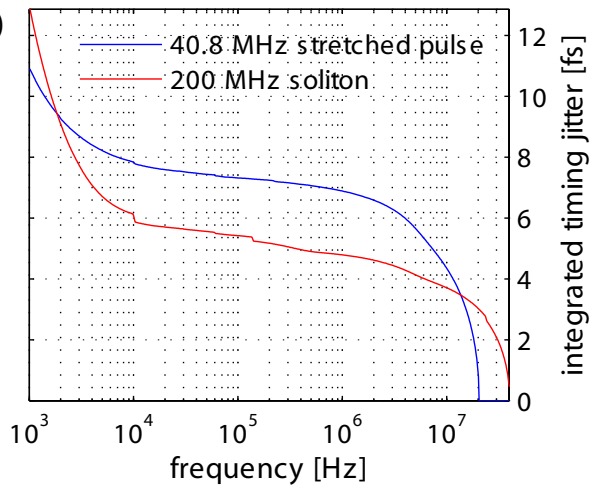

Fig. 1. Phase noise of a $40.8 \mathrm{MHz}$ stretched-pulse laser and a $200 \mathrm{MHz}$ soliton laser. a) Single-sideband phase noise measured at the harmonics at $980 \mathrm{MHz}$ (stretched-pulse) and $1.2 \mathrm{GHz}$ (soliton), respectively. b) Integrated timing jitter starting integration at $20.4 \mathrm{MHz}$ (stretched-pulse) and $40 \mathrm{MHz}$ (soliton).

The pulse train generated by this laser is distributed to the remote locations via actively length-stabilized, dispersion compensated fiber links [3]. At the end stations, the light pulses serve different purposes: they are converted into RF signals [4] which are needed in the accelerating cavities, they are used to synchronize external lasers by optical crosscorrelation or seeding, or they are used directly for opto-diagnostic devices [5, 6]. A synchronization branch from the 
fiber laser to the remote end-station requires multiple amplification stages in order to keep the average laser power in a suitable range. An important task is, therefore, a careful characterization of the influence of the EDFAs on the performance of the synchronization system.

A setup (see Fig. 2 a)) based on a balanced optical cross-correlator [7] was used to measure the timing jitter added by an EDFA with sub-femtosecond resolution. The $100 \mathrm{fs}$ long laser pulses of a $200 \mathrm{MHz}$ soliton laser are amplified by a counter-pumped, dispersion compensated EDFA.
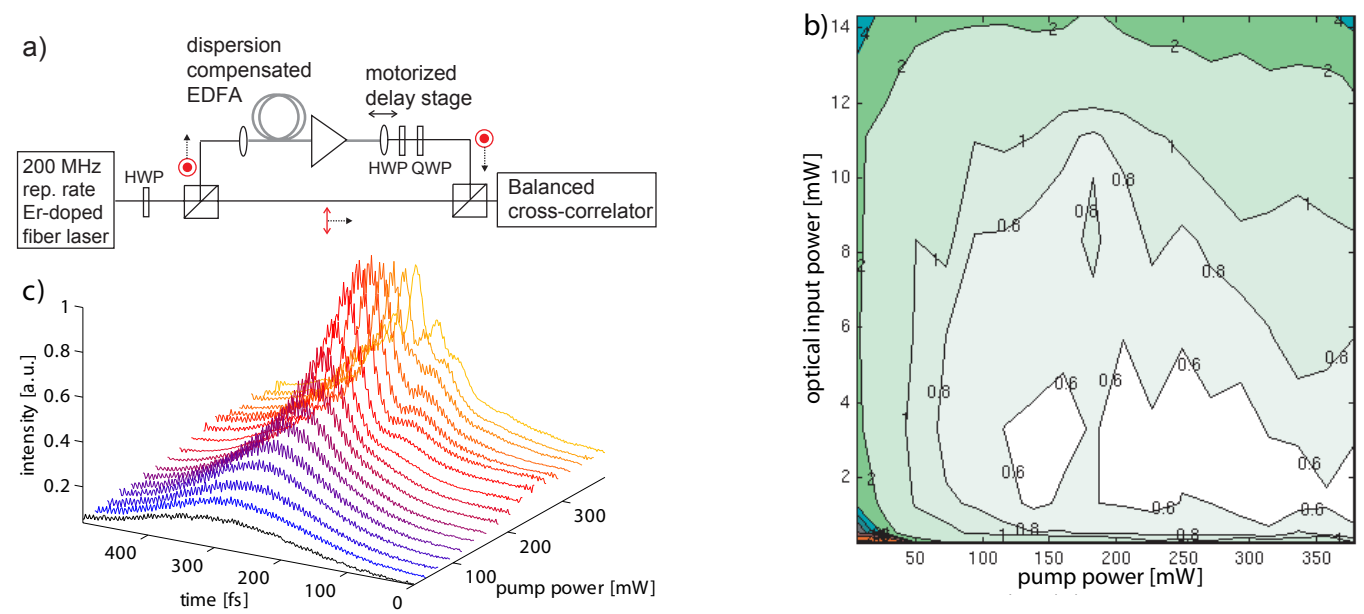

Fig. 2. a) Experimental setup for the timing jitter characterization caused by EDFAs. b) Integrated timing jitter ( $500 \mathrm{~Hz}-4.5 \mathrm{MHz})$ in femtoseconds added by the EDFA as a function of the optical input power and the power of the $980 \mathrm{~nm}$ pump light. c) auto-correlation traces of amplified laser pulses for different pump powers (input power: $14.3 \mathrm{~mW}$ )

The timing jitter contribution of the EDFA was measured as a function of the optical input power and the power of the $980 \mathrm{~nm}$ pump light. A new calibration of the detector was performed for every set of parameters, since the response of the cross-correlator depends on the intensity and pulse length of the incoming laser pulses. Figure $2 \mathrm{~b}$ ) shows the integrated timing jitter $(500 \mathrm{~Hz}-4.5 \mathrm{MHz})$ added by the EDFA. For a weakly pumped EDFA and optical input powers below $1 \mathrm{~mW}$ the added timing jitter reaches up to $40 \mathrm{fs}$. Under optimized working conditions, the added timing jitter is below $0.5 \mathrm{fs}(\mathrm{rms})$, measured with a resolution of around $0.1 \mathrm{fs}$.

For weak pump powers the gain fiber is not entirely saturated and hence, absorption of the pulse train occurs. For large amplifications the output pulse energies reaches up to $0.5 \mathrm{~nJ}$ (100 $\mathrm{mW}$ output power). At these pulse energies, the laser pulses are distorted due to nonlinearities inside the optical fiber (see Fig. 2 c)) and intensity noise on the optical pulse train is converted into changes of the pulse shape.

In summary, we found that the characterization of the EDFAs used within the synchronization system is crucial for system performance. Systematic measurements of the timing jitter added by the amplification to a pulse train from an EDFA are presented. Depending on pump power settings, the timing jitter varies between $0.5 \mathrm{fs}$ to $40 \mathrm{fs}$. The measurement principle applied in this work can also be used to characterize the influence of other components on the timing stability of optical pulse trains.

\section{References}

1. J. Kim et. al., "Large scale timing distribution and RF-synchronization for FEL facilities," FEL Conference 2004, Trieste, Italy, 2004.

2. J. Chen et. al., Opt. Lett. 32, 1566-1568 (2007).

3. F. Loehl et. al., "First Tests of fiber-link Stabilization Based on Optical Cross-Correlation for the FLASH Synchronization System," DIPAC Conference 2007, Venice, Italy, 2007.

4. J. Kim, F. Ludwig, M. Felber, and F. X. Kärtner, Opt. Express 15, 8951 (2007).

5. F. Loehl et. al., "A Sub-50 fs Bunch Arrival Time Monitor System for FLASH," DIPAC Conference 2007, Venice, Italy, 2007.

6. K. Hacker et. al., "Large Horizontal Aperture BPM and Precision Bunch Arrival Pickup”, DIPAC Conference 2007, Venice, Italy, 2007.

7. J. Kim et. al., Opt. Lett. 32, 1044-1046 (2007). 